\title{
PHISHING-PT: BASE DE DADOS EM PORTUGUÊS PARA FILTRAGEM AUTOMÁTICA DE PHISHING EM E-MAIL
}

\author{
Lucas Scaravelli, Kenzo Ikeizume, Rodrigo Nogueira e Johannes Lochter \\ Departamento de Engenharia de Computação, \\ Centro Universitário Facens, Brasil
}

\begin{abstract}
RESUMO
O aumento da utilização da Internet tornou popular a realização de transações bancárias e comerciais de forma online, tornando o ambiente da Internet atrativo e interessante para fraudadores. Uma das principais técnicas para aplicar fraude é a técnica de phishing, que utiliza engenharia social para enganar o usuário com uma mensagem que parece legítima, atraindo-o para uma página falsa ao clicar em algum hyperlink. Ao ser direcionado para a página falsa, o usuário acaba cedendo dados sensíveis como credenciais de acesso e dados pessoais aos fraudadores. Como as técnicas de phishing evoluem muito rapidamente, é importante que sejam estudadas e criadas técnicas de filtragem automática que auxiliem os usuários a não serem enganados. Devido aos ataques de phishing utilizarem linguagem natural e normalmente serem feitos no idioma do usuário almejado como alvo, é importante que se tenha referência de outros idiomas além do inglês, língua em que normalmente as pesquisas são feitas nesse tópico. Esse artigo traz como contribuição uma nova base de dados de phishing em português, os resultados de filtragem automática utilizando métodos de classificação clássicos da literatura e uma discussão rica apontando que, embora todos os métodos apresentem bons resultados, o método Multinomial Naive Bayes tem a menor taxa de filtragem incorreta de mensagens fraudulentas como mensagens legítimas.
\end{abstract}

\section{PALAVRAS-CHAVE}

Filtragem de Phishing, Segurança da Informação, Processamento de Linguagem Natural, Inteligência Artificial

\section{INTRODUÇÃO}

A cada ano aumenta o número de pessoas que usufruem da Internet conforme divulgação feita pelas organizações Hootsuite e We Are Social, a qual aponta que mais de 4 bilhões de pessoas no mundo todo utilizam a Internet. Por conta do aumento do número de internautas, a produção de dados e armazenamento de informações na rede também aumentaram, conforme apontado pela consultoria da IDC (International Data Corporation), levando a uma expectativa de que a quantidade de informação armazenada dobrará anualmente até 2025, chegando a 175 zettabytes de dados (Patrizio, 2018).

No Brasil, as aplicações que manipulam informações pessoais (bancos, cartões de crédito ou seguradoras) são obrigadas pela lei a seguir um padrão de segurança elaboradas pelo PCI (Security Standards Council), um fórum global aberto que determina padrões de segurança para a proteção de dados, como por exemplo: não usar padrões disponibilizados pelo fornecedor para senhas do sistema e outros parâmetros de segurança; criptografar a transmissão de dados do titular do cartão em redes abertas e públicas; e testar regularmente os sistemas e processos de segurança.

Mesmo que as empresas e órgãos públicos garantam a integridade das informações, grupos criminosos especializados em fraudes acessam esses dados através de outras maneiras. Na maioria das vezes, o caminho mais difícil é usurpar informações por meio de hacking. Geralmente denominadas como cracker (um hacker mal-intencionado), essas pessoas devem possuir alto conhecimento na área de redes e programação para executar suas ações.

Por ser um meio mais acessível, um dos métodos mais empregado por grupos mal-intencionados é o phishing: a expressão originária do verbo pescar em inglês, tem como objetivo "pescar" dados de usuários desatentos através de e-mails, mensagens de texto e sites falsos. Os fraudadores ludibriam o usuário, fazendo-o ceder seus dados propositalmente (Chiew, 2018). 
Os assuntos abordados nos casos de phishing geralmente tentam atingir o emocional dos usuários como, por exemplo, vagas de emprego, liberação de benefícios ou isenção de impostos. Também há uma grande quantidade de páginas falsas em que o objetivo é simular uma operação de um determinado banco, como por exemplo: recadastramento, desbloqueio de cartões ou recuperação de conta. O objetivo é fazer o cliente da instituição fornecer os dados como login, senha, CPF ou cartão de crédito de forma natural, induzindo-o acreditar que é um ambiente seguro. Um exemplo de phishing é dado na Figura 1, onde o usuário é induzido a acreditar que se trata de uma mensagem legítima do banco e, ao clicar em "Recadastramento" acabará fornecendo dados bancários sensíveis a terceiros mal-intencionados.

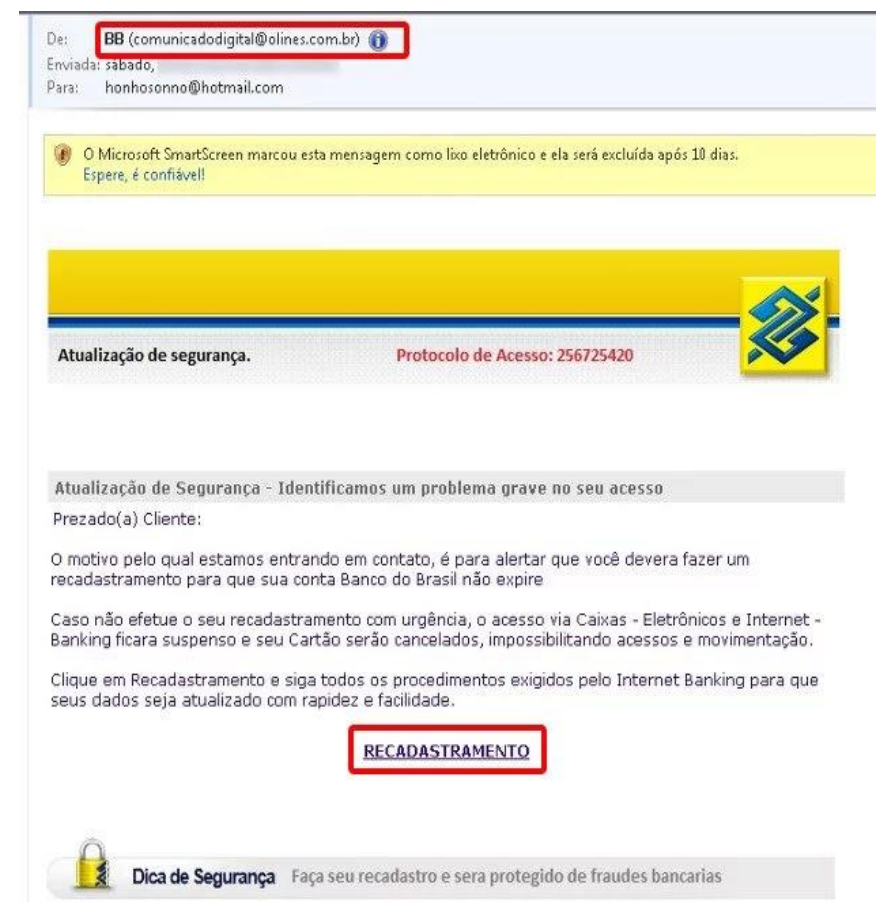

Figura 1. Exemplo de phishing para enganar usuários a fim de obter-se dados bancários

Do lado dos bancos e empresas prejudicadas, a busca é constante por meios de combater essas ações e evitar prejuízos e frustrações de seus clientes. Hoje são utilizados canais de comunicação, como centrais telefônicas e atendimento particular nas agências, para agilizar as providências após algum cliente perceber que seus dados foram expostos. Muitas vezes esses métodos são pouco eficientes devido a velocidade de ação dos fraudadores. Como alternativa, as empresas e os centros de pesquisa começaram a estudar maneiras de aplicar o reconhecimento automático de phishing para evitar que o usuário seja atraído para armadilha.

Esse artigo é fruto de um trabalho de conclusão de curso para titulação em Engenharia de Computação e suas principais contribuições são: (1) uma base de dados nova a respeito de phishing em português; (2) resultados de métodos clássicos da literatura aplicados na base de dados proposta e (3) discussão entre os métodos apontando qual método apresenta melhor taxa de acerto considerando também a menor taxa de falsos positivos, ou seja, phishing classificados como legítimos, que levariam o usuário a clicar em mensagens mal-intencionadas.

\section{TRABALHOS RELACIONADOS}

Segundo estudo da empresa de segurança digital McAfee, o Brasil perde mais de 10 bilhões de dólares com crimes cibernéticos. A pesquisa ainda aponta que 54\% dos ataques praticados no Brasil originam do próprio território com conteúdo fraudulento redigido em português para atingir os usuários (Machado, 2018). 
As técnicas de aprendizagem automática combinadas ao processamento de linguagem natural podem ser importantes ferramentas para filtrar automaticamente links maliciosos e mensagens fraudulentas entregues pelos golpes de phishing (Chiew, 2018; Peng, 2018).

Em processamento de linguagem natural, as mensagens de texto são representadas numericamente em uma etapa de pré-processamento e, em seguida, são processadas por um algoritmo de classificação que baseado no conteúdo da amostra, poderá indicar se o conteúdo é legítimo e confiável, ou fraudulento e malicioso (Almeida, 2011).

Em (Sahingoz, 2019), os autores avaliaram a eficácia de aplicar a filtragem automática de phishing na identificação de links maliciosos. Utilizando o método de florestas aleatórias e trabalhando a engenharia de atributos, os autores reportaram 97,98\% de acurácia na solução do problema. Em comparação ao trabalho citado, esse artigo traz uma análise mais profunda com relação às mensagens fraudulentas incorretamente classificadas como legítimas e utiliza o corpo de mensagens de e-mail em vez de links.

Uma pesquisa mais ampla conduzida recentemente (Chiew, 2018) demonstrou que o assunto de filtragem automática de phishing se tornou recorrente na literatura. Enquanto há muitos tipos de phishing e para cada tipo um conjunto de técnicas diferentes que enfrentam o problema da filtragem automática, esse artigo foca no phishing disseminado por mensagens de e-mail e emprega a representação bag-of-words para transformar as amostras de texto em informação numérica para o processamento das amostras pelos métodos de classificação.

A representação computacional bag-of-words (Harris, 1954) transforma a amostra de texto em um vetor numérico do tamanho do vocabulário previamente coletado, o qual identifica de forma única cada palavra ou termo diferente no corpo de texto. Cada posição do vetor é alimentada com a frequência ou ocorrência do elemento do vocabulário daquele índice na amostra em questão. Enquanto há técnicas mais modernas de representar as amostras de texto de maneira numérica, essa técnica ainda apresenta resultados no estado-da-arte e é aplicada ao problema de phishing (Chiew, 2018).

A maioria dos trabalhos e pesquisas conduzidas com phishing são feitos no idioma inglês, inviabilizando a criação de filtros automáticos para outros idiomas. Após extensivas buscas, e no que cerne o conhecimento dos autores, não há uma base pública de mensagens de e-mail voltadas para a tarefa de filtragem automática de phishing, portanto também não há trabalhos relacionados com o assunto e nenhuma comparação nesse sentido será feita por conta da restrição encontrada. Esse trabalho apresenta então, uma base inédita para uso futuro e resultados de baseline para efeito de comparação também.

\section{METODOLOGIA}

As amostras que compõem o conjunto de dados proposto neste artigo foram originadas de dois principais meios: os e-mails legítimos foram adquiridos através de um formulário online proposto pelos autores (Figura 2) e os e-mails foram obtidos de forma colaborativa, enquanto os e-mails de phishing foram extraídos do catálogo online da Rede Nacional de Ensino e Pesquisa (RNP, 2018). As mensagens legítimas foram coletadas de forma colaborativa e tratadas para evitar o vazamento de dados sensíveis, impossibilitando a identificação dos usuários cedentes da informação. A coleta foi feita em um intervalo de aproximadamente três meses, entre fevereiro de 2019 e abril de 2019, totalizando 257 e-mails legítimos e 799 e-mails fraudulentos.

A partir da base de dados coletada foram simulados três cenários que representam três diferentes situações para avaliar o desempenho dos métodos de classificação de aprendizagem automática em cada cenário:

A. Todas as amostras coletadas, mantendo o desbalanceamento original da coleta;

B. Mensagens selecionadas com temas em comum: transações bancárias, orçamentos, compras online e promoções;

C. De acordo com um relatório da empresa Kapersky (2014), as mensagens fraudulentas representam $0,0071 \%$ de todas mensagens de e-mails trocadas no mundo. Para manter essa proporção, seria necessária uma coleta inicial de 14.084 amostras legítimas. Nesse sentido, os autores deste artigo propuseram o desbalanceamento em torno de $13 \%$ para representar o cenário mais próximo do desbalanceamento apontado no relatório. 


\section{Insira seu e-mail aqui}

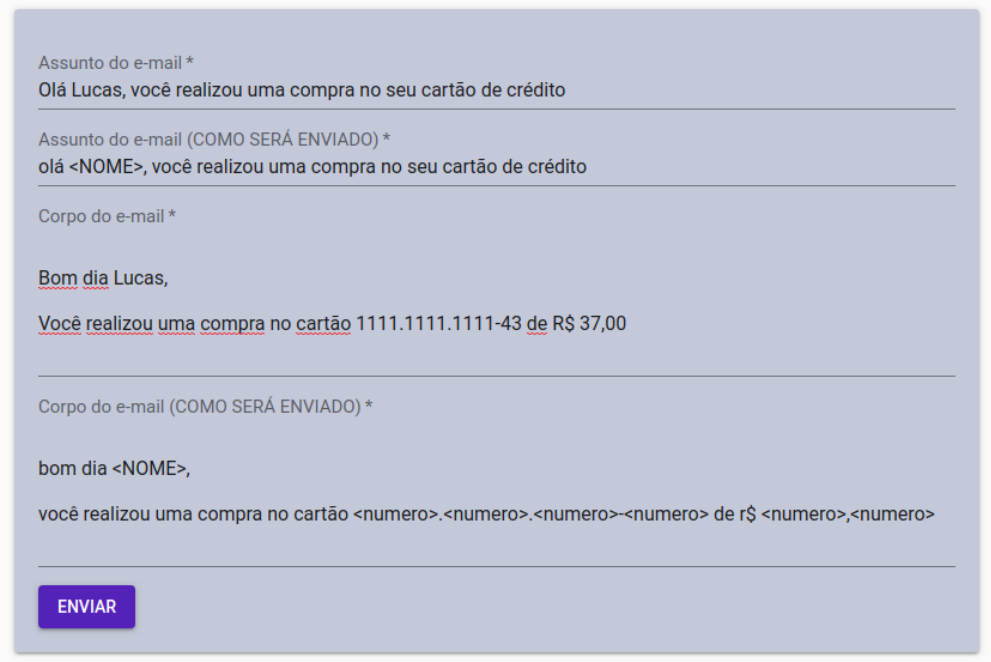

Figura 2. Formulário disponibilizado para preenchimento colaborativo com amostras de mensagens legítimas

Os cenários e as estatísticas de quantidade e proporção entre mensagens legítimas e fraudulentas (phishing) são apresentados na Tabela 1 .

Tabela 1. Cenários de avaliação de detecção automática de phishing

\begin{tabular}{lllll}
\hline Identificação & Amostras legítimas & Amostras de phishing & Proporção phishing para total & Total de amostras \\
\hline A & 257 & 799 & $75 \%$ & 1056 \\
B & 95 & 95 & $50 \%$ & 190 \\
C & 200 & 30 & $13 \%$ & 230 \\
\hline
\end{tabular}

Os autores procuraram variar e avaliar métodos de classificação baseados em diferentes estratégias de seleção de hipótese, como probabilísticos, regras de decisão, vizinhança e otimização. Os métodos de classificação escolhidos para apresentar os resultados preliminares na base de dados proposta foram escolhidos tomando como base trabalhos na literatura semelhantes e considerados relevantes de acordo com os canais de publicação em que foram veiculados, como periódicos e congressos com alto fator de impacto e número de citações (Sahingoz, 2018; Peng, 2018).

A partir dos métodos de classificação foi realizado uma busca em grade ( grid search) para encontrar os melhores parâmetros mediante cada cenário, utilizando validação cruzada de 5 partições. Os melhores parâmetros encontrados por método de classificação para cada cenário avaliado são apresentados na Tabela 2.

Os resultados são apresentados e analisados a partir das métricas extraídas das matrizes de confusão da execução de cada método de classificação. As mensagens legítimas corretamente classificadas são identificadas como (VL - verdadeiro legítima), as mensagens legítimas incorretamente classificadas como phishing são identificadas como (FL - falso legítima), assim como as mensagens de phishing corretamente e incorretamente classificadas são identificadas respectivamente como VP e FP. As métricas utilizadas para avaliar os métodos de classificação nesse artigo são descritas e formuladas na Tabela 3. 
Tabela 2. Melhores parâmetros por cenário e método de classificação de phishing

\begin{tabular}{lll}
\hline Cenário & Método de classificação & Melhores parâmetros \\
\hline A & Multinomial Naive Bayes & alpha $=0,1$ \\
& Bernoulli Naive Bayes & alpha $=0,01$ \\
& N-Vizinhos Próximos & $\mathrm{n}=3$ \\
& Regressão Logística & $\mathrm{C}=1000 ;$ penalidade $=\mathrm{L} 2$ \\
& Árvores de Decisão & divisão da amostra $=2$ \\
& SVM & $\mathrm{C}=1 ;$ kernel $=$ linear \\
\hline Multinomial Naive Bayes & alpha $=0,01$ \\
& Bernoulli Naive Bayes & alpha $=0,001$ \\
& N-Vizinhos Próximos & $\mathrm{n}=11$ \\
& Regressão Logística & $\mathrm{C}=0,01 ;$ penalidade $=\mathrm{L} 2$ \\
& Árvores de Decisão & divisão da amostra $=2$ \\
& SVM & $\mathrm{C}=10 ;$ kernel = linear \\
\hline M & Multinomial Naive Bayes & alpha $=0,01$ \\
& Bernoulli Naive Bayes & alpha $=0,1$ \\
& N-Vizinhos Próximos & $\mathrm{n}=5$ \\
& Regressão Logística & $\mathrm{C}=100 ;$ penalidade $=\mathrm{L} 1$ \\
& Árvores de Decisão & divisão da amostra $=2$ \\
& SVM & $\mathrm{C}=1 ;$ kernel $=$ linear \\
\hline
\end{tabular}

Tabela 3. Métricas utilizadas para avaliar o desempenho dos métodos de classificação

\begin{tabular}{lll}
\hline Métrica & Legítima (L) & Phishing (P) \\
\hline Precisão (Pr) & VL / (VL + FL) & VP / (VP + FP) \\
Revocação (Re) & VL / (VL + FP) & VP / (VP + FL) \\
Acurácia (Ac) & $(\mathrm{VL}+\mathrm{VP}) /(\mathrm{VL}+\mathrm{VP}+\mathrm{FL}+\mathrm{FP})$ \\
F-Medida (Fm) & $2 *[(\mathrm{Pr} \mathrm{P} * \mathrm{Re} \mathrm{P}) /(\mathrm{Pr} \mathrm{P}+\mathrm{Re} \mathrm{P})]$ \\
\hline
\end{tabular}

\section{RESULTADOS PRELIMINARES}

A execução dos métodos de classificação nos diferentes cenários considerando os melhores parâmetros encontrados respectivamente são apresentados nas Tabelas 3, 4 e 5 e discutidos a seguir. Os valores em negrito por coluna indicam o maior índice encontrado para a respectiva métrica, descrita na coluna em questão.

Tabela 4. Resultados dos métodos de classificação para o cenário A

\begin{tabular}{lcccccc}
\hline Método de classificação & F-Medida & Acurácia & $\begin{array}{c}\text { Precisão } \\
\text { (Legítimo) }\end{array}$ & $\begin{array}{c}\text { Revocação } \\
\text { (Legítimo) }\end{array}$ & $\begin{array}{c}\text { Precisão } \\
\text { (Phishing) }\end{array}$ & $\begin{array}{c}\text { Revocação } \\
\text { (Phishing) }\end{array}$ \\
\hline Multinomial Naive Bayes & $96,58 \%$ & $88,28 \%$ & $\mathbf{9 8 , 2 9 \%}$ & $54,24 \%$ & $86,67 \%$ & $\mathbf{9 9 , 6 8 \%}$ \\
Bernoulli Naive Bayes & $\mathbf{9 7 , 0 7 \%}$ & $\mathbf{8 9 , 4 6 \%}$ & $97,67 \%$ & $\mathbf{5 9 , 4 3 \%}$ & $\mathbf{8 7 , 9 8 \%}$ & $99,52 \%$ \\
N-Vizinhos Próximos & $88,40 \%$ & $75,26 \%$ & $66,66 \%$ & $2,83 \%$ & $75,35 \%$ & $99,52 \%$ \\
Regressão Logística & $96,55 \%$ & $85,20 \%$ & $95,78 \%$ & $42,92 \%$ & $83,86 \%$ & $99,63 \%$ \\
Árvores de Decisão & $89,98 \%$ & $77,27 \%$ & $57,93 \%$ & $34,43 \%$ & $80,66 \%$ & $91,62 \%$ \\
SVM & $96,24 \%$ & $87,33 \%$ & $98,16 \%$ & $50,47 \%$ & $85,73 \%$ & $\mathbf{9 9 , 6 8 \%}$ \\
\hline
\end{tabular}

Utilizando todas as amostras da base de dados proposta, os resultados apresentados na Tabela 3 têm como objetivo apresentar o baseline para futuras comparações de métodos e técnicas envolvendo a aplicação dessa base de dados. O método Bernoulli Naive Bayes apresentou o maior índice de F-Medida (97,07\%), a qual representa a média harmônica da precisão e revocação de phishing. É possível perceber que os métodos Multinomial Naive Bayes, Regressão Logística e SVM tiveram resultados semelhantes, enquanto os métodos Árvore de decisão e N-Vizinhos Próximos ficaram abaixo (8\% abaixo em média).

Entre os melhores métodos em termo de F-Medida também é importante as métricas de Precisão (Legítimo) e Revocação (Phishing) porque traçam a relação de mensagens fraudulentas classificadas como mensagens legítimas. Esse erro de classificação poderia levar o usuário a clicar em uma mensagem fraudulenta que foi filtrada como legítima, podendo levar à perda de dados sensíveis e credenciais de acesso. Nesse sentido, o 
método Bernoulli Naive Bayes apresenta o maior índice de F-Medida, mas o método Multinomial Naive Bayes além de apresentar índice de F-Medida muito próximo ao método Bernoulli, também apresenta índices melhores de Precisão Legítimo (98,29\%) e Revocação Phishing (99,68\%). Baseada nessa análise, o melhor método para um cenário semelhante ao cenário A seria o método Multinomial Naive Bayes.

Tabela 5. Resultados dos métodos de classificação para o cenário B

\begin{tabular}{lcccccc}
\hline Método de classificação & F-Medida & Acurácia & $\begin{array}{c}\text { Precisão } \\
\text { (Legítimo) }\end{array}$ & $\begin{array}{c}\text { Revocação } \\
\text { (Legítimo) }\end{array}$ & $\begin{array}{c}\text { Precisão } \\
\text { (Phishing) }\end{array}$ & $\begin{array}{c}\text { Revocação } \\
\text { (Phishing) }\end{array}$ \\
\hline Multinomial Naive Bayes & $\mathbf{8 9 , 5 1 \%}$ & $\mathbf{6 5 , 7 8 \%}$ & $60,55 \%$ & $88,00 \%$ & $44,15 \%$ & $56,66 \%$ \\
Bernoulli Naive Bayes & $88,26 \%$ & $\mathbf{7 7 , 6 3 \%}$ & $\mathbf{7 2 , 0 4 \%}$ & $89,33 \%$ & $66,23 \%$ & $\mathbf{7 5 , 0 0 \%}$ \\
N-Vizinhos Próximos & $81,94 \%$ & $59,86 \%$ & $56,60 \%$ & $80,00 \%$ & $40,25 \%$ & $50,40 \%$ \\
Regressão Logística & $85,10 \%$ & $55,26 \%$ & $52,44 \%$ & $\mathbf{1 0 0 , 0 0 \%}$ & $11,68 \%$ & $20,93 \%$ \\
Árvores de Decisão & $74,98 \%$ & $59,86 \%$ & $69,44 \%$ & $33,33 \%$ & $\mathbf{8 5 , 7 1 \%}$ & $68,39 \%$ \\
SVM & $85,30 \%$ & $63,81 \%$ & $58,47 \%$ & $92,00 \%$ & $50,45 \%$ & $50,45 \%$ \\
\hline
\end{tabular}

Para o cenário $\mathrm{B}$, onde os dados estão balanceados na mesma quantidade de amostras por classe, os resultados apresentados na Tabela 5 mostram que o maior índice de F-Medida foi obtido utilizando o método de classificação Multinomial Naive Bayes. No entanto, considerando a análise feita para o cenário A, esse método apresenta índices menores para Precisão (Legítimos) e Revocação (Phishing). Isso indica que o método filtra incorretamente muitas mensagens fraudulentas e mostra para o usuário essas mensagens como legítimas.

Alternativas ao método Multinomial é o método Bernoulli, pois tem F-Medida próxima $(88,26 \%)$ ao melhor resultado, mas apresenta taxas maiores de Precisão Legítimo (72,04\% se comparado a 60,55\% do método Multinomial) e Revocação Phishing (75\% se comparado a 56,66\% do método Multinomial). Nesse cenário, onde a proporção de mensagens legítimas e phishing é balanceada, é aconselhável a utilização do método Bernoulli, em vez do método Multinomial.

Tabela 6. Resultados dos métodos de classificação para o cenário C

\begin{tabular}{lcccccc}
\hline Método de classificação & F-Medida & Acurácia & $\begin{array}{c}\text { Precisão } \\
\text { (Legítimo) }\end{array}$ & $\begin{array}{c}\text { Revocação } \\
\text { (Legítimo) }\end{array}$ & $\begin{array}{c}\text { Precisão } \\
\text { (Phishing) }\end{array}$ & $\begin{array}{c}\text { Revocação } \\
\text { (Phishing) }\end{array}$ \\
\hline Multinomial Naive Bayes & $\mathbf{6 9 , 5 4 \%}$ & $87,57 \%$ & $89,26 \%$ & $97,08 \%$ & $66,66 \%$ & $\mathbf{3 3 , 3 3 \%}$ \\
Bernoulli Naive Bayes & $63,45 \%$ & $\mathbf{8 9 , 4 4 \%}$ & $\mathbf{8 9 , 4 7 \%}$ & $99,27 \%$ & $88,88 \%$ & $\mathbf{3 3 , 3 3 \%}$ \\
N-Vizinhos Próximos & $59,07 \%$ & $85,09 \%$ & $85,09 \%$ & $\mathbf{1 0 0 , 0 0 \%}$ & $0,00 \%$ & $0,00 \%$ \\
Regressão Logística & $67,51 \%$ & $86,95 \%$ & $86,70 \%$ & $\mathbf{1 0 0 , 0 0 \%}$ & $\mathbf{1 0 0 , 0 0 \%}$ & $12,50 \%$ \\
Árvores de Decisão & $46,25 \%$ & $88,81 \%$ & $88,38 \%$ & $\mathbf{1 0 0 , 0 0 \%}$ & $\mathbf{1 0 0 , 0 0 \%}$ & $25,00 \%$ \\
SVM & $42,66 \%$ & $87,57 \%$ & $87,26 \%$ & $\mathbf{1 0 0 , 0 0 \%}$ & $\mathbf{1 0 0 , 0 0 \%}$ & $16,66 \%$ \\
\hline
\end{tabular}

Finalmente, os resultados referentes à execução dos métodos de classificação considerando o cenário C são apresentados na Tabela 6 . O cenário C é caracterizado pela proporção de phishing muito pequena com relação à quantidade de amostras legítimas presentes na base. Para esse cenário, o método Multinomial Naive Bayes teve o melhor índice de F-Medida (69,54\%), seguido do método Regressão Logística (67, 51\%), enquanto os outros métodos apresentaram resultados inferiores em, no mínimo, 4 pontos percentuais. Devido ao método Multinomial ter tido a segunda melhor Precisão (Legítimos), perdendo apenas para o método Bernoulli (89,44\%) e empatando no índice de Revocação (Phishing), o método Multinomial é o mais aconselhável para esse cenário.

Na comparação entre os cenários foi notado que o método Multinomial apresentou os melhores índices de F-Medida entre os métodos avaliados. Complementar a esse resultado, os maiores índices da métrica Revocação (Phishing) para os maiores índices de F-Medida também foram obtidos pelo método Multinomial, cuja métrica aponta a qualidade do método em classificar corretamente as mensagens de phishing sem expor usuário ao perigo confundindo mensagens de phishing como mensagens legítimas. Esses resultados mostram que o método Multinomial teve desempenho satisfatório para a tarefa proposta independente do balanceamento dos cenários testados, tornando-se o método aconselhável entre os métodos clássicos avaliados para filtragem automática de phishing. 


\section{CONCLUSÃO E DIREÇÕES FUTURAS}

O presente trabalho propõe a utilização de técnicas de aprendizado de máquina para fazer a filtragem de mensagens de e-mail de phishing a fim de evitar que o usuário seja enganado por mensagens fraudulentas e perca dados sensíveis, como credenciais de acesso. Além disso, a principal contribuição é uma base de phishing em português, visto que a maioria das pesquisas nessa área se destinam ao idioma inglês e os ataques de phishing acontecem normalmente no idioma das vítimas.

Os experimentos foram conduzidos considerando três cenários com balanceamento diferente entre as classes mensagens legítimas e mensagens fraudulentas: um cenário considerando todas as mensagens da base, um cenário considerando proporção igual entre as classes e um cenário extremamente desbalanceado com poucas amostras de phishing. Após a análise dos resultados preliminares, foi observado que o método mais indicado é o Multinomial Naive Bayes independente do cenário, pois além de apresentar índice alto de F-Medida, apresentou os maiores valores de revocação na classe phishing, cuja métrica aponta a qualidade do método em não confundir mensagens de phishing com mensagens legítimas.

A expectativa de conclusão desse trabalho envolve novos passos de avaliação de técnicas utilizando representação distribuída vetorial, onde cada palavra passa a ser representada por um vetor com características sintáticas e semânticas capturadas a partir do processamento de um grande corpo de texto. Esse tipo de representação também favorecerá a utilização de técnicas mais atuais para processamento de linguagem natural como redes neurais recorrentes, as quais são impossibilitadas pela técnica de representação bag-of-words apresentada neste artigo, devido à alta dimensionalidade e esparsidade. Como última etapa, também está previsto a implementação de um filtro para a plataforma Gmail que identifica phishing automaticamente mediante aprovação de acesso pelo usuário responsável por uma conta de e-mail.

\section{REFERENCIAS}

Almeida, T. A. et al, 2011. Redução de dimensionalidade aplicada na classificação de spams usando filtros bayesianos. In Revista Brasileira de Computação Aplicada, Vol. 3, No. 1, pp.16-29

Chiew, K. L. et al, 2018. A survey of phishing attacks: Their types, vectors and technical approaches, In Expert Systems with Applications, Vol. 106, No. 1, pp 1-20.

Harris, Z., 1954. Distributional Structure. In Word, Vol. 10, No.2, pp 146-162.

Machado, F., 2018. Brasil perde US\$ 10 bilhões por ano com cibercrime, diz McAfee, acessado em 20 Setembro 2019, $<$ https://veja.abril.com.br/economia/brasil-perde-us-10-bilhoes-por-ano-com-cibercrime-diz-mcafee/>.

Patrizio, A., 2018. IDC: Expect 175 zettabytes of data worldwide by 2025, Network World, acessado em 20 Setembro 2019, < https://www.networkworld.com/article/3325397/idc-expect-175-zettabytes-of-data-worldwide-by2025.html>.

Peng, T. et al, 2018. Detecting phishing attacks using natural language processing and machine learning. In IEEE 12th international conference on semantic computing, pp. 300-301.

RNP, 2018. Fraudes identificadas e divulgadas pelo CAIS, acessado em 20 Setembro 2019, <http://memoria.rnp.br/cais/fraudes.php?tag_extend=\&tag=92>.

Sahingoz, O. K et al, 2019. Machine learning based phishing detection from URLs, In Expert Systems with Applications, Vol. 117, No. 1, pp. 345-357. 